Jondon, S.W.1, and at Church House, Dean's Yard, Westminster, London, S.W.1. Following the week of lectures, there will be tours and day excursions to research stations, gardens, nurseries, fruit- and seedgrowing establishments, etc.; visits to places of historical interest will also be included. Other events that will take place during the Congress are as follows. The International Committee for Horticultural Congresses will meet on September 7 and 13; the International Committee on Horticultural Nomenclature and Registration will meet on September 7 ; a meeting of representatives of botanic gardens under the auspices of the International Botanic Ciardens Commission will be held on September 13 ; and the Flower Show of the Royal Horticultural Society will take place during September 9-10. The membership fee for the Congress is 4 guineas. Further details can be obtained from the Secretary, Thirteenth International Horticultural Congress, Royal Horticultural Society, Vincent Square, London, S.W.1.

\section{The Potsdam Geodetic Institute}

Following on the political changes in Germany resulting from the War, the Potsdam Geodetic Institute has renewed the publication of its work in a new series of Veröffentlichungen, of which six issues have been received, of dates 1949-51. They appear under the auspices of the Deutsche Akademie der Wissenschaften zu Berlin, of which the Geodetic Institute became in 1946 a dependent body, under the directorship of F. Mühlig. 'The Institute welcomes exchanges of its publications with those of other geodetic workers. The six issues now available deal partly with work begun before the War, as in the study (No. 2, F. Mühlig) of the 24-metre interference comparator, the construction of which was begun in 1937, and in No. 3 (K. Weiken), dealing with pendulum observations, 1934-43. Nos. 1 and 6, by K. Reicheneder, deal with the theory of errors and adjustment of networks in nadir triangulations; No. 4, by H. Haalck, is on gravity-disturbed fields and on the theory of the normal figure of the earth; and No. 5, by the late K. Friedrich and W. Jenne, discusses in much detail the solutions of systems of linear equations in which many coefficients are zero. The back covers of the later issues list some of the post-war publications of the Potsdam Ceophysical Institute, prepared by R. Bock, F. Burmeister, F. Errulat, and W. Schumann; they deal entirely with geomagnetism.

\section{Jodrell Bank Annals: A New Astronomical Journal}

A NEW publication, to be known as the Jodrell Bank Annals, initiates Series 1 of the Astronomical Contributions from the University of Manchester (Vol. 1, No. 1, pp. 1-12; 1952). Through this medium a considerable amount of observational data will be made available to astronomers, especially in connexion with the continuous operation of the meteor, solar and galactic noise equipments at the Jodrell Bank Experimental Station. It is hoped, however, that the Annals will also contain, from time to time, contributions to theoretical astronomy from the University and its Mathematical Laboratory. The first publication, "Radio. Echo Observations of the Daytime Meteor Streams in 1951", by G. S. Hawkins and Mary Almond, includes a certain amount of material that has previously appeared in Mon. Not. Roy. Astro. Soc. and also in Nature; but a few new features are worth attention. A new minor stream was detected near $\cup$ Piscium on May 12 and 13 ; but the radiant structure was diffuse. Although it is suggested that this stream may correspond to the Piscid streams observed in 1947 and 1948, the difference of $10^{\circ}$ in the right ascension of the radiant renders the identification uncertain. The $\eta$ Aquarid stream supplied meteors with velocities $63 \cdot 7,63 \cdot 0$ and $58.8 \mathrm{~km}$. $/ \mathrm{sec}$., respectively, as shown in Table 4 ; but meteors radiating from a radiant at R.A. $336^{\circ}$, Dec. $0^{\circ}$, with a period of Halley's Comet, should have a velocity of $70.4 \mathrm{~km}$./sec. (velocities in all cases are geocentric), and the velocities indicated by the radio echo method correspond to a period of about ten years. This implies that the stream cannot be closely aligned with the orbit of Halley's Comet, nor could it be associated with the October Orionids. Some astronomers doubt very much the connexion of Halley's Comet with either stream-and in particular the Orionids-and hold that the apparent similarity in orbital elements is probably fortuitous. In Mon. Not. Roy. Astro. Soc., 111, 37 (1951), it was shown by Mary Almond that the $\zeta$ Perseids and the night-time S. Arietids on October 12 , radiant at R.A. $38^{\circ}$, Dec. $12^{\circ}$, were probably associated, and the work of 1951 has reaffirmed the connexion. We can confidently anticipate many interesting items in this new publication.

\section{British Library of Political and Economic Science}

THE annual report for $1950-51$ of the British Library of Political and Economic Science (pp. 22 ; London School of Fconomics, 1951) refers to restrictions on expenditure necessitated by the heavy expenditure of the previous year and the increased cost of books and periodicals, but acquisitions during the year brought the total of bound volumes to 323,575 as against 315,185 in 1950 , of which 11,281 were in the lending library and 3,891 in the Shaw library. Expenditure on treatises was $£ 4,187$, as against $£ 5,377$ in $1949-50$, and on periodicals $£ 5,524$ as against $£ 3,711$; and 6,707 periodicals were currently received as against 6,291 in the previous year. Treatises issued, however, fell from 44,969 to 37,669 ; but works lent to other libraries increased from 516 to 761 , while research readers' permits increased from 452 to 482 . Progress has been made with the preparation of the three further volumes of the "London Bibliography of the Social Sciences" which will cover the intake of the Library during 1936-50. A special grant permitted the clearing-off of most of the arrears of binding which had accumulated since the War, but extensions to the fabric had again to be postponed. A list of publications by members of the Library staff is appended.

\section{British Mammals}

ThE Trustees of the British Museum (Natural History) have again put naturalists in their debt by the production of "A List of British Mammals" (2s. net). This has been written by T. C. S. MorrisonScott who, after an explanatory account of how the scientific names of animals are devised, describes the use of certain keys to the external characters and skulls of those species which might confuse the layman. Among the mammals described are the hedgehog, mole, seven species of shrew, twelve species of bat, the fox, pine marten and three species of stoat, the weasel, two species of polecat, badger, otter, Scottish wild cat, seven species of deer, three species of hare, the rabbit, two species of dormouse, eighteen species of field mouse, the black and brown 